Research Article

\title{
The Analysis on Seepage Field of Grouted and Shotcrete Lined Underwater Tunnel
}

\author{
Zhenggui Qin $\mathbb{D}^{1,2}$ Yuan Wang ${ }^{1},{ }^{1}$ Yi Song, ${ }^{3}$ and Qi Dong ${ }^{1}$ \\ ${ }^{1}$ College of Civil and Transportation Engineering, Hohai University, 1 Xikang Road, Nanjing 210098, China \\ ${ }^{2}$ China Design Group Co., LTD., \\ Research and Development Center of Transport Industry of Technologies and Equipments for Intelligent Design, \\ Construction and Maintenance of Underwater Tunnel, Ministry of Transport, Nanjing 210098, China \\ ${ }^{3}$ China Railway Liyuan Survey \& Design Group Co., LTD., Tianjin 300308, China \\ Correspondence should be addressed to Zhenggui Qin; qzghhu@outlook.com
}

Received 8 October 2019; Accepted 25 February 2020; Published 5 May 2020

Academic Editor: Alessandro Mauro

Copyright $(2020$ Zhenggui Qin et al. This is an open access article distributed under the Creative Commons Attribution License, which permits unrestricted use, distribution, and reproduction in any medium, provided the original work is properly cited.

Groundwater control in underwater tunnels by drilling and blasting method is generally carried out by grouting ring, shotcrete lining (primary lining), and concrete lining. The permeable grouting ring and shotcrete lining have an important impact on seepage field. However, the currently published research models of related results are for homogeneous and isotropic single-layer unlined tunnels, ignoring the important effects of tunnel grouting circles and primary lining. If the conclusions of the relevant literature are directly used to guide the tunnel design, large errors may occur. Therefore, on the basis of previous studies, this article extends the tunnel seepage research model and incorporates the tunnel grouting ring and primary lining into the research model. The research model is more in line with actual working conditions. Based on the principle of mirror method, the seepage field of a drainage tunnel in an infinite aquifer is superposed with that of a water supply tunnel in an infinite aquifer, and the analytical solution to the seepage field of an grouted and shotcrete lined underwater tunnel in a semi-infinite aquifer is obtained, which is further verified by numerical analysis and experiment. In addition, the influence of grouting ring and primary lining parameters on seepage field is discussed by using partial differential analysis. The results show that the seepage flow of tunnel can be significantly alleviated by either reducing the permeability coefficient of grouting ring and primary lining or increasing the thickness of grouting ring and primary lining, but the water pressure of grouting ring and primary lining will increase.

\section{Introduction}

Underwater tunnels play an important role in modern traffic engineering. The well-known examples include Seikan Tunnel (1988), English Channel Tunnel (1993), and Submarine Tunnel of Hong Kong-Zhuhai-Macao Bridge (2018). However, a large amount of water gushing in tunnels, on one hand, can cause serious environmental problems; on the other hand, they will cause huge drainage costs for $\mathrm{V}$-shaped tunnels. The water pressure around the tunnel is related to the safety and durability of the tunnel structure. Therefore, groundwater control technology is particularly critical among many key technical problems that must be solved in underwater tunnel engineering.

Since the early 1960s, the study of tunnel seepage has attracted much attention, and scholars have done a lot of work on it. Harr [1] initially proposed an image method to obtain the pore pressure and inflow into a unlined tunnel in a homogeneous, isotropic, and semi-infinite aquifer, and this method was employed by many other researchers Lei [2]. Goodman [3] further developed a simplified approximation formula, which is widely used at present. Zhang and Franklin [4] extended the solution which took into account the varying hydraulic conductivity of medium. El Tani $[5,6]$ derived an equation for exact gravity water inflow into a circular tunnel based on Mobius-transformation and Fourier series. Kolymbas and Wagner et al. [2, 7] presented an inflow formula for both deep and shallow tunnels. Park et al. [8-10] compared two existing analytical solutions based on conformal mapping with different boundary conditions along the tunnel circumference (one with zero water pressure and the other with a constant hydraulic head). Yang and Yeh [11] provided a closed-form solution to estimate the flow rate of 
the groundwater into a tunnel during the progressive drilling in a multilayer formation system. Fernandez and Moon [12] provided an analytical method for estimating the groundwater inflow into a tunnel in which the lowered groundwater level after excavation is adopted. Jordi et al. (2011) implemented a method for predicting groundwater inflows at tunnel face scale. Marechal et al. [13] developed an analytical solution for modeling discharge into a tunnel drilled in a heterogeneous unconfined aquifer. Maréchal et al. [14] developed a semianalytical method to predict groundwater tunnel inflow considering both the initial water level and the lowered water level. Ying et al. Su [15] derived analytical solutions for pore pressure, seepage force, and water ingress into a subaqueous drained tunnel with a circular section in a homogenous isotropic semi-infinite seabed considering the effect of tide. Zareifard [16] presented an analytical solution for analysis and design of pressure tunnels, based on a generalized effective stress principle. In addition, some empirical and numerical models were provided to evaluate groundwater flow into tunnel, e.g., [12, 17-23]. Summarily, existing approximation solutions of the groundwater inflow are provided in Table $1[24,25]$.

Drilling and blasting tunnels are widely constructed according to the concept of NATM. They are composed of grouting ring, shotcrete lining (primary lining), and concrete lining. Groundwater control is generally designed according to the principle of "prevention first, limited discharge." That is to say, part of groundwater is allowed to infiltrate into the tunnel through drainage system and then discharged into the tunnel through drainage system such as ditches. Therefore, there is a relatively stable seepage field in the operation period.

Obviously, the permeable grouting ring and the primary lining are important components of the tunnel seepage field and have an important impact on the tunnel seepage field. It can be seen from Table 1 that the related published research results and models studied are for homogeneous and isotropic single-layer unlined tunnels, ignoring the important effects of tunnel grouting circles and primary lining. If the conclusions of the relevant literature are directly used to guide the tunnel design, large errors may occur. Therefore, on the basis of previous studies, this article extends the tunnel seepage research model and incorporates the tunnel grouting ring and primary lining into the research model. The research model is more in line with actual working conditions. In specific, based on the principle of mirror method, the seepage field of a drainage tunnel in an infinite aquifer stratum and a water supply tunnel in an infinite aquifer stratum are superimposed to obtain the seepage field of an underwater tunnel in accordance with the actual working conditions. The accuracy of the theoretical solution further is verified by numerical analysis and experiments. The conclusions obtained by different methods are consistent. The influence of grouting ring and primary lining parameters on seepage field is also discussed. The conclusions of this paper can be considered as a reference for the design of underwater tunnels.

The structure of this paper is as follows: the first section of the text introduces the tunnel model and the principle of mirror method, the second section details the process of analytical analysis, the third section introduces the situation of numerical simulation verification, the fourth section describes the situation of experimental verification, the fifth section further discusses some important issues, and the sixth section briefly summarizes the conclusion.

\section{Models and Methods}

2.1. Tunnel Seepage Model. In order to simplify the problem properly, the model studied in this paper is based on the four assumptions as follows:

(i) The underwater tunnel is circular and located in homogeneous and isotropic surrounding rock.

(ii) The tunnel structure is composed of shotcrete lining and concrete lining, with surrounding rock grouting ring outside the shotcrete lining.

(iii) Concrete lining is a nonpermeable structure and is not included in seepage study. The inner side of shotcrete lining is fixed head boundary.

(iv) The seepage field is stable and conforms to Darcy's law.

The tunnel model is shown in Figure 1. The inner radius of shotcrete lining is $r_{0}$, the outer radius of shotcrete lining (equal to the inner radius of grouting ring) is $r_{l}$, the outer radius of grouting ring is $r_{g}$, the water depth is $d_{1}$, the burial depth below the riverbed (or seabed) in the center of tunnel is $d_{2}$, and the permeability coefficients of shotcrete lining, grouting ring, and stratum are $k_{l}, k_{g}$, and $k_{s}$, respectively. The inner water head of shotcrete lining is $H_{0}$, and the water head at the ground surface in is $H_{R}$.

2.2. The Principle of Mirror Method. The semi-infinite boundary seepage problem mentioned above is rather complicated, and it can be simplified by using the principle of mirror method. The tunnel is regarded as a complete well in a composite confined aquifer, and the riverbed (or seabed) is taken as the symmetrical axis. On the opposite side of the tunnel, it is assumed that there is a virtual tunnel with equal recharge and tunnel drainage (the structure of the virtual tunnel is exactly the same as that of the real tunnel). The seepage field of the virtual tunnel and the real tunnel in the infinite aquifer is superimposed. The actual tunnel seepage field in the semi-infinite aquifer is obtained, as shown in Figure 2.

\section{Analysis and Deduction}

According to the principle of mirror method mentioned above, the tunnel is regarded as a complete well, and the stable seepage field of a complete well in an infinite confined aquifer is the basis for subsequent analysis. The following is the first analysis.

3.1. Stable Seepage Field of Complete Well without Lining in Infinite Confined Aquifer. The stable seepage field of a 
TABLE 1: Approximation solutions of groundwater inflow into unlined nongrouting tunnel.

\begin{tabular}{|c|c|c|}
\hline Literature & Formula & Description \\
\hline Harr [1] & $Q_{H a}=2 \pi k\left(h / \ln \left(r_{B l} / r_{A l}\right)\right)$ & $\begin{array}{c}\text { Initial water level, deep tunnels, homogeneous, isotropic, } \\
\text { and semi-infinite aquifer }\end{array}$ \\
\hline Goodman [3] & $Q_{G o}=2 \pi k(h / \ln (2 h / r))$ & $\begin{array}{c}\text { Initial water level, deep tunnels, homogeneous, isotropic, } \\
\text { and semi-infinite aquifer }\end{array}$ \\
\hline Zhang and Franklin & $Q_{Z F}=2 \pi k\left(h / \ln \sqrt{1+\left(4 h^{2} / r^{2}\right)}\right)$ & $\begin{array}{l}\text { Initial water level, varying hydraulic conductivity of } \\
\text { medium in jointed rock deep tunnels }\end{array}$ \\
\hline $\begin{array}{l}\text { Kolymbas and } \\
\text { Wagner }[2,7]\end{array}$ & $Q_{L K}=2 \pi k\left(h / \ln \left((h / r)+\sqrt{\left(h^{2} / r^{2}\right)-1}\right)\right)$ & Initial water level, for both deep and shallow tunnels \\
\hline El Tani $[5]$ & $Q_{E l}=2 \pi k h\left(1-3(r / h)^{2}\right) /\left[1-(r / 2 h)^{2}\right] \ln (2 h / r)-(r / 2 h)^{2}$ & $\begin{array}{l}\text { Initial water level, tunnels of circular, elliptical, or square } \\
\text { cross sections, nonhomogeneous aquifer }\end{array}$ \\
\hline Karlsrud (2001) & $Q_{K a}=2 \pi k(h / \ln ((2 h / r)-1))$ & $\begin{array}{c}\text { Initial water level, tunnel, homogeneous, isotropic, and } \\
\text { semi-infinite aquifer }\end{array}$ \\
\hline $\begin{array}{l}\text { Fernandez and } \\
\text { Moon [12] }\end{array}$ & $\begin{array}{c}Q_{M F 1}=k\left(2 R_{y} h-h^{2}\right) / R_{x}-r \text { (shallow tunnel) } \\
Q_{M F 2}=2 \pi k(\bar{h} / \ln (2 h / r)) \text { (deep tunnel) }\end{array}$ & $\begin{array}{l}\text { Lowered water level, using permeability reduction of } \\
\text { medium, for both deep and shallow tunnels }\end{array}$ \\
\hline
\end{tabular}

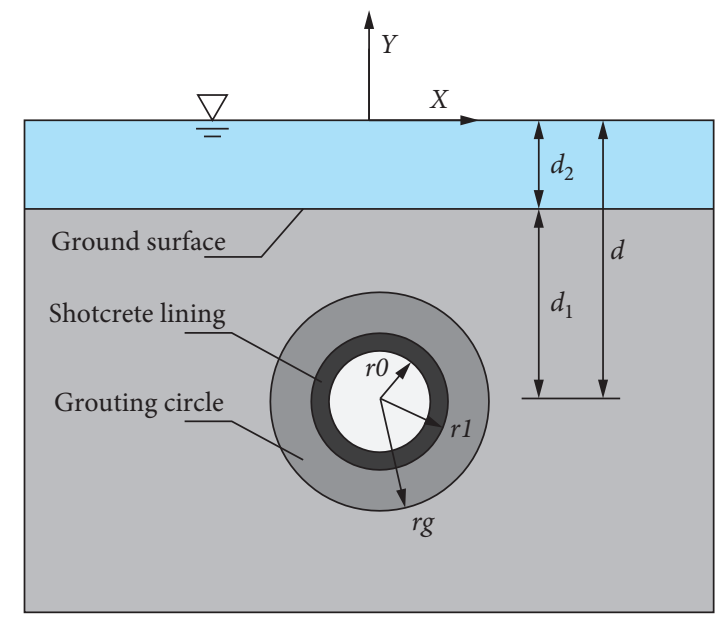

FIgURE 1: The seepage model of underwater tunnel.
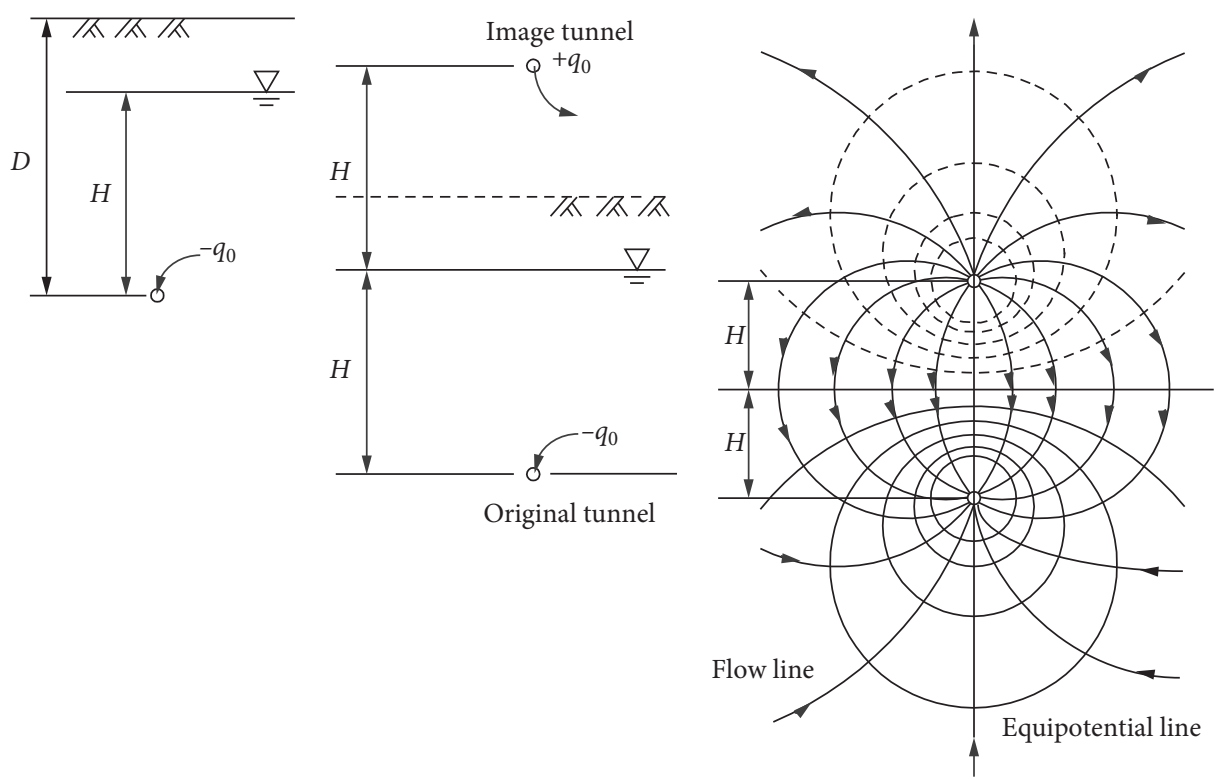

(a)

(b)

(c)

FIGURE 2: The principle of mirror method [12]. 


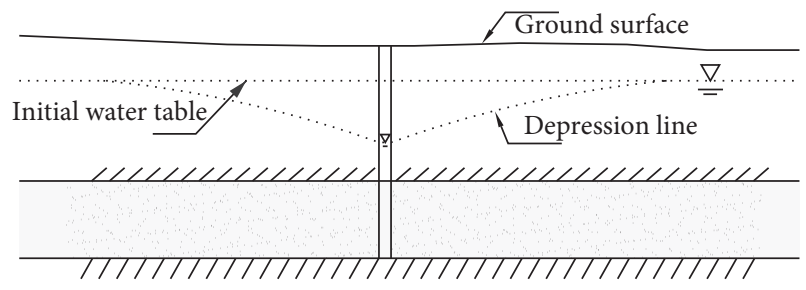

(a)

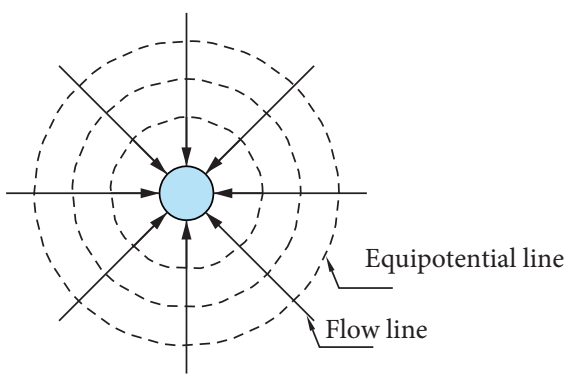

(b)

Figure 3: Stable seepage field of complete well without lining.

complete well without lining in a homogeneous and isotropic confined aquifer (as shown in Figure 3) has the following two characteristics:

(1) Seepage is a horizontal radiation flow, i.e., streamlines are uniformly radioactive and converge in the center of the well. The isopotential surface of seepage is a cylindrical surface centered on the well axis.

(2) The seepage flow through each equipotential surface is the same and equal to the water inflow of the well.

The head potential function of the above seepage field satisfies the Laplace equation and can be expressed in cylindrical coordinates as follows [1]:

$$
\nabla^{2} \phi=\frac{1}{r} \frac{\partial}{\partial r}\left(r \frac{\partial \phi}{\partial r}\right)+\frac{1}{r^{2}} \frac{\partial \phi^{2}}{\partial \theta^{2}}=0 .
$$

Since the seepage field is axisymmetric and independent of angle, it can be simplified as

$$
\frac{1}{r} \frac{\partial}{\partial r}\left(r \frac{\partial \phi}{\partial r}\right)=0
$$

Its general understanding is as follows:

$$
\left\{\begin{array}{l}
\phi=\frac{q}{2 \pi} \ln r+C, \\
\phi=k H .
\end{array}\right.
$$

\subsection{Stable Seepage Field of Multilayer Structure Well in Infinite} Confined Aquifer. When the confined well is a multilayer structure (as shown in Figure 4), i.e., centering on the well axis, the multilayer structure is cylindrical from inside to outside, and the medium of each layer is homogeneous and isotropic, the distribution law of seepage field is the same as that of the complete well without lining in the confined aquifer described in the preceding section, but the potential function in the medium of each layer is different, which can be expressed as follows:

$$
\left\{\begin{array}{l}
\phi_{i}=\frac{q}{2 \pi} \ln r+C_{i}, \\
\phi_{i}=k_{i} H .
\end{array}\right.
$$

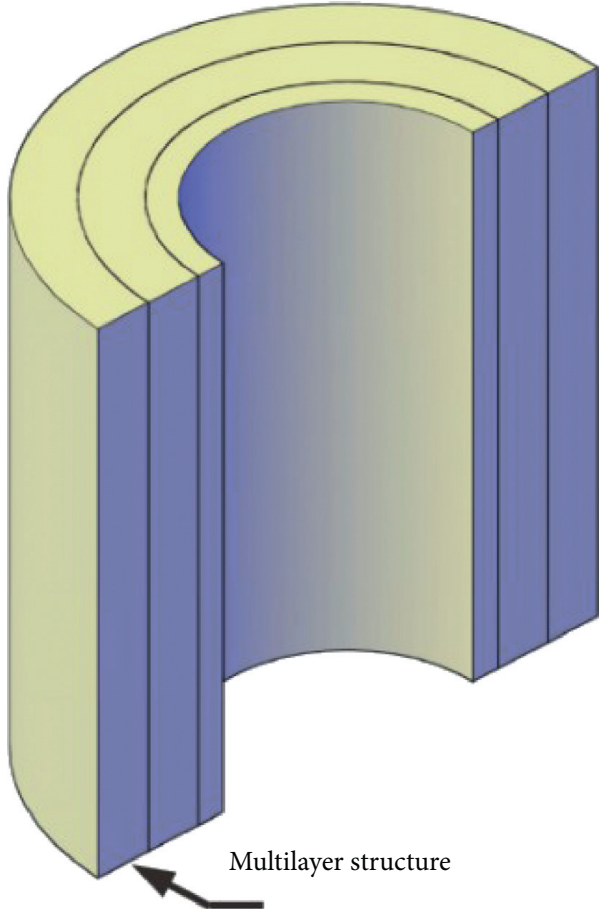

Figure 4: Multilayer structure well.

3.3. Stable Seepage Field of Multilayer Structure Tunnel in Infinite Aquifer. Compared with Figures 1 and 4, it can be seen that a tunnel with shotcrete lining and grouting ring in an infinite aquifer can be regarded as a complete well with double-layer structure, and its potential function is rewritten as follows:

$$
\phi_{\sin k}= \begin{cases}\phi_{A 1}=\frac{-q}{2 \pi} \ln r_{A}+C_{A 1}=-k_{l} H, & \left(r_{A} \leq r_{l}\right), \\ \phi_{A 2}=\frac{-q}{2 \pi} \ln r_{A}+C_{A 2}=-k_{g} H, & \left(r_{l} \leq r_{A} \leq r_{g}\right), \\ \phi_{A 3}=\frac{-q}{2 \pi} \ln r_{A}+C_{A 3}=-k_{s} H, & \left(r_{g} \leq r_{A}\right) .\end{cases}
$$

The virtual tunnel in the infinite aquifer is a recharge tunnel, and its seepage is positive. Its potential function is as follows: 


$$
\phi_{\text {source }}= \begin{cases}\phi_{B 1}=\frac{q}{2 \pi} \ln r_{B}+C_{B 1}=-k_{l} H, & \left(r_{B} \leq r_{l}\right), \\ \phi_{B 2}=\frac{q}{2 \pi} \ln r_{B}+C_{B 2}=-k_{g} H, & \left(r_{l} \leq r_{B} \leq r_{g}\right), \\ \phi_{B 3}=\frac{q}{2 \pi} \ln r_{B}+C_{B 3}=-k_{s} H, & \left(r_{g} \leq r_{B}\right) .\end{cases}
$$

3.4. Stable Seepage Field of Multilayer Tunnel in Semi-Infinite Aquifer. According to the principle of mirror method, the potential function of multilayer structure tunnel in semiinfinite aquifer is as follows:

$$
\phi=\phi_{\text {sin } k}+\phi_{\text {source }}
$$

The formulas (5) and (6) are superimposed,

$$
\phi= \begin{cases}\phi_{1}=\phi_{A 1}+\phi_{B 1}=\frac{q}{2 \pi} \ln \frac{r_{B}}{r_{A}}+C_{1}=-k_{l} H, & \left(r_{A} \leq r_{l}\right), \\ \phi_{2}=\phi_{A 2}+\phi_{B 2}=\frac{q}{2 \pi} \ln \frac{r_{B}}{r_{A}}+C_{2}=-k_{g} H, & \left(r_{l} \leq r_{A} \leq r_{g}\right), \\ \phi_{3}=\phi_{A 3}+\phi_{B 3}=\frac{q}{2 \pi} \ln \frac{r_{B}}{r_{A}}+C_{3}=-k_{s} H, & \left(r_{g} \leq r_{A}\right) .\end{cases}
$$
there are

On the inner boundary of the tunnel shotcrete lining,

$$
\phi_{\Omega 0}=\frac{q}{2 \pi} \ln \frac{r_{B 0}}{r_{A 0}}+C_{1}=-k_{l} H_{0} .
$$

On the riverbed (or seabed), there are

$$
\phi_{\Omega_{R}}=\frac{q}{2 \pi} \ln \frac{r_{B R}}{r_{A R}}+C_{3}=-k_{s} H_{R} .
$$

On the contact surface between shotcrete lining and grouting ring, there are

$$
\left\{\begin{array}{l}
\phi_{\Omega l}=\frac{q}{2 \pi} \ln \frac{r_{B l}}{r_{A l}}+C_{1}=-k_{l} H_{l}, \\
\phi_{\Omega l}=\frac{q}{2 \pi} \ln \frac{r_{B l}}{r_{A l}}+C_{2}=-k_{g} H_{l} .
\end{array}\right.
$$

On the contact surface between grouting circle and formation, there are

$$
\left\{\begin{array}{l}
\phi_{\Omega g}=\frac{q}{2 \pi} \ln \frac{r_{B g}}{r_{A g}}+C_{2}=-k_{g} H_{g}, \\
\phi_{\Omega g}=\frac{q}{2 \pi} \ln \frac{r_{B g}}{r_{A g}}+C_{3}=-k_{s} H_{g} .
\end{array}\right.
$$

To solve (8)-(12) equations, there are

$$
\left\{\begin{array}{l}
q=\frac{2 \pi k_{l} k_{g} k_{s}\left(H_{R}-H_{0}\right)}{k_{g} k_{s} \ln \left(r_{B 0} r_{A l} / r_{A 0} r_{B l}\right)+k_{l} k_{s} \ln \left(r_{B l} r_{A g} / r_{A l} r_{B g}\right)+k_{l} k_{g} \ln \left(r_{B g} / r_{A g}\right)}, \\
C_{1}=-k_{l} H_{0}-\frac{q}{2 \pi} \ln \frac{r_{B 0}}{r_{A 0}}, \\
C_{2}=\left(\frac{q}{2 \pi k_{l}} \ln \frac{r_{A 0} r_{B l}}{r_{B 0} r_{A l}}+\frac{q}{2 \pi k_{g}} \ln \frac{r_{A l}}{r_{B l}}-H_{0}\right) k_{g}, \\
C_{3}=-k_{s} H_{R}-\frac{q}{2 \pi} \ln \frac{r_{B R}}{r_{A R}} .
\end{array}\right.
$$

The head distribution function of seepage field is obtained by substituting (13) for (8),

$$
H= \begin{cases}H_{1}=H_{0}+\frac{q}{2 \pi k_{l}} \ln \frac{r_{B 0} r_{A}}{r_{A 0} r_{B}}, & \left(r_{A} \leq r_{l}\right), \\ H_{2}=H_{0}+\frac{q}{2 \pi k_{l}} \ln \frac{r_{B 0} r_{A l}}{r_{A 0} r_{B l}}+\frac{q}{2 \pi k_{g}} \ln \frac{r_{B l} r_{A}}{r_{A l} r_{B}}, & \left(r_{l} \leq r_{A} \leq r_{g}\right), \\ H_{3}=H_{R}-\frac{q}{2 \pi k_{s}} \ln \frac{r_{B} r_{A R}}{r_{A} r_{B R}}, & \left(r_{g} \leq r_{A}\right) .\end{cases}
$$

According to the relationship between water head and water pressure $P=(H-y) \times \gamma_{w}$, water pressure in seepage field can be obtained by substituting water head function,

$$
P= \begin{cases}P_{1}=\left(H_{0}+\frac{q}{2 \pi k_{l}} \ln \frac{r_{B 0} r_{A}}{r_{A 0} r_{B}}-y\right) \times \gamma_{w}, & \left(r_{A} \leq r_{l}\right), \\ P_{2}=\left(H_{0}+\frac{q}{2 \pi k_{l}} \ln \frac{r_{B 0} r_{A l}}{r_{A 0} r_{B l}}+\frac{q}{2 \pi k_{g}} \ln \frac{r_{B l} r_{A}}{r_{A l} r_{B}}-y\right) \times \gamma_{w}, & \left(r_{l} \leq r_{A} \leq r_{g}\right), \\ P_{3}=\left(H_{R}-\frac{q}{2 \pi k_{s}} \ln \frac{r_{B} r_{A R}}{r_{A} r_{B R}}-y\right) \times \gamma_{w}, & \left(r_{g} \leq r_{A}\right) .\end{cases}
$$




\section{Validation of Numerical Simulation}

In order to verify the accuracy of the above analysis, the seepage field of a typical underwater drilling and blasting tunnel under various working conditions is analyzed by numerical simulation.

4.1. Numerical Simulation Model. Assuming a typical underwater tunnel, of which values of parameters used are listed in Table 2, and four cases may exist as follows.

Case I: the tunnel with none of shotcrete lining and grouting circle; Case II: the tunnel with a grouting circle; Case III: the tunnel with a shotcrete lining; Case IV: the tunnel with both the shotcrete lining and grouting circle.

4.2. Numerical Simulation Results. The water inflow into tunnel in various cases is given in Table 3 . The results show that the solution derived in this paper is very close to the ABAQUS numerical solution.

The water head distribution in cases is given in Figures 5-8.

The comparison of water pressure between the solution presented in this paper and ABAQUS numerical solution are shown in Figures 9-12. Obviously, they show good agreement.

\section{Test Verification}

5.1. Testing Device. As shown in Figure 13, according to the ratio of $1: 100$, a test box is made with the size of $0.55 \mathrm{~m} \times 0.60 \mathrm{~m} \times 0.60 \mathrm{~m}$, the diameter of the simulated tunnel is $5.5 \mathrm{~cm}$, and the thickness of the grouting ring is $4.5 \mathrm{~cm}$. Fixed water head boundary is applied in the flume on both sides of the box, and white geotextile is used to prevent soil particles from losing; 12 pressure measuring tubes are arranged above the box to observe the water head at each point, of which 0 points are used to observe the water pressure at the boundary of the tunnel, 6, 7, 8, and 9 points are used to observe the water pressure behind the grouting ring, and the rest points are used to observe the surrounding rock medium water pressure.

5.2. Test Setting. As shown in Figure 14, the first step is to place the sleeve of the simulated tunnel in the center and fix it well.

The second step is to layered fill coarse sand outside the sleeve to simulate the surrounding rock. The measured permeability coefficient is $8.5 \times 10^{-5} \mathrm{~m} / \mathrm{s}$.

The third step is to fill the sleeve with fine sand and simulate the grouting ring. The measured permeability coefficient is $5 \times 10^{-5} \mathrm{~m} / \mathrm{s}$.

In the fourth part, the cover plate is sealed and the box body is positioned.

5.3. Test Process. The head of $1.13 \mathrm{~m}$ was applied to the flume on both sides of the tank and remained unchanged during the test. When the whole box has been saturated, keep the
TABle 2: Parameters value of a typical underwater tunnel.

\begin{tabular}{lc}
\hline Variable & Value $(\mathrm{m})$ \\
\hline$r_{0}$ & 5 \\
$r_{l}$ & 5.3 \\
$r_{g}$ & 10 \\
$d_{1}$ & 5 \\
$d_{2}$ & 30 \\
$H_{R}$ & 5 \\
$H_{0}$ & -30 \\
Variable & Value $(\mathrm{m} / \mathrm{s})$ \\
$K_{l}$ & $1 \times 10^{-8}$ \\
$K_{g}$ & $1 \times 10^{-7}$ \\
$K_{s}$ & $2 \times 10^{-6}$ \\
\hline
\end{tabular}

Table 3: Comparison of water inflow into tunnel.

\begin{tabular}{lccc}
\hline Water inflow & Cases & This proposed method & ABAQUS \\
\hline \multirow{3}{*}{$Q\left(\mathrm{~m}^{3} / \mathrm{d}\right)$} & I & 15.30 & 15.10 \\
& II & 2.44 & 2.36 \\
& III & 2.70 & 2.62 \\
& IV & 1.46 & 1.41 \\
\hline
\end{tabular}

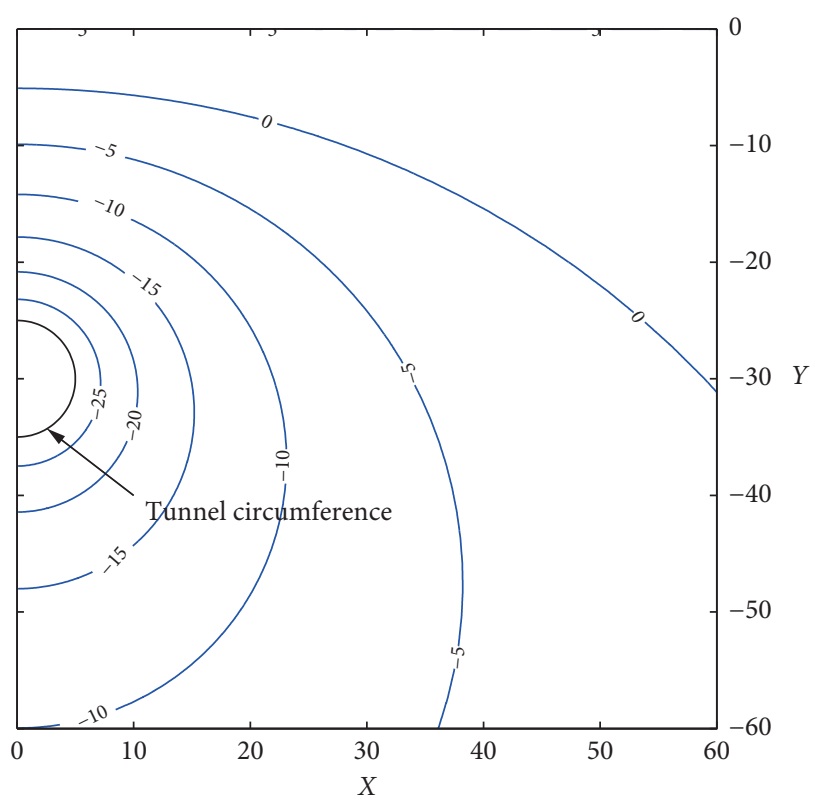

FIgURE 5: The water head distribution in case I.

tunnel outlet closed and observe the distribution of water head of each piezometric pipe. If the water head equals the total water head applied on both sides, it shows that the piezometric pipe is smooth and effective, and the test can be started, as shown in Figure 15.

Experiments and observations under four working conditions have been carried out successively, as follows:

Working condition 1: Keep the pore pressure of measuring point 6 at $6.6 \mathrm{kpa}$, measure the steady flow rate of outlet and pore pressure of other measuring points.

Working condition 2: Keep the pore pressure of measuring point 6 at $5.5 \mathrm{kpa}$, measure the steady flow rate of the outlet and pore pressure of other measuring points. 


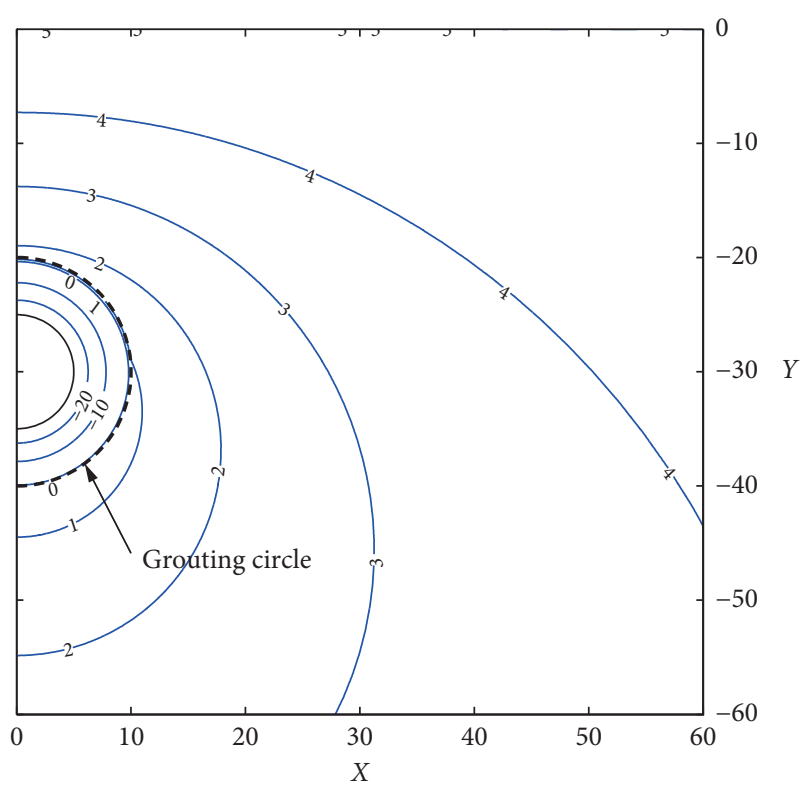

FIGURE 6: The water head distribution in case II.

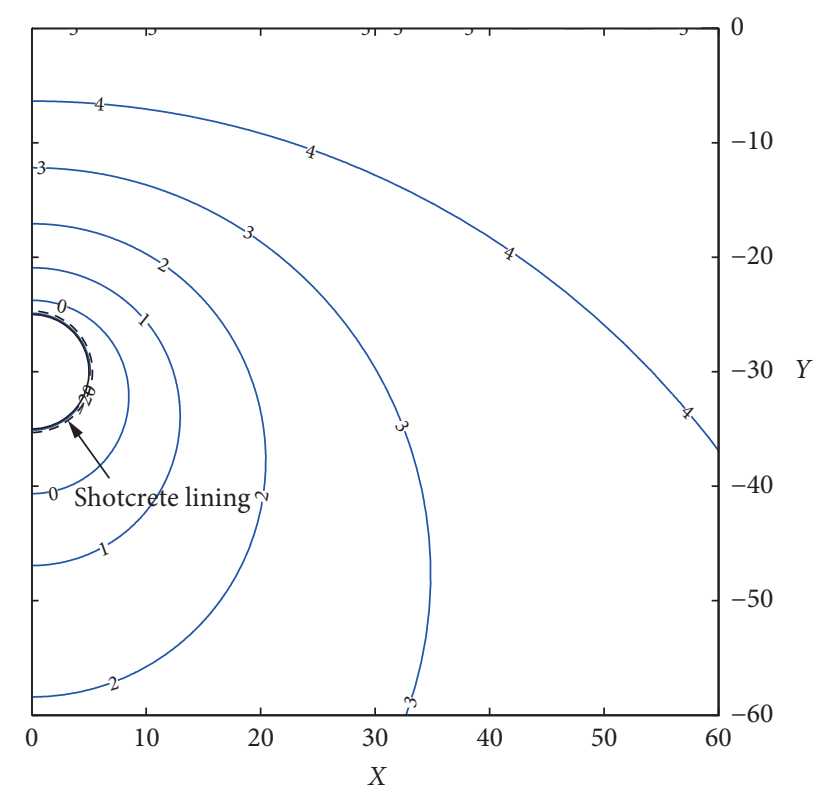

FIgURE 7: The water head distribution in case III.

Working condition 3: Keep the pore pressure of measuring point 6 at $4.5 \mathrm{kpa}$, measure the steady flow rate of the outlet and pore pressure of other measuring points.

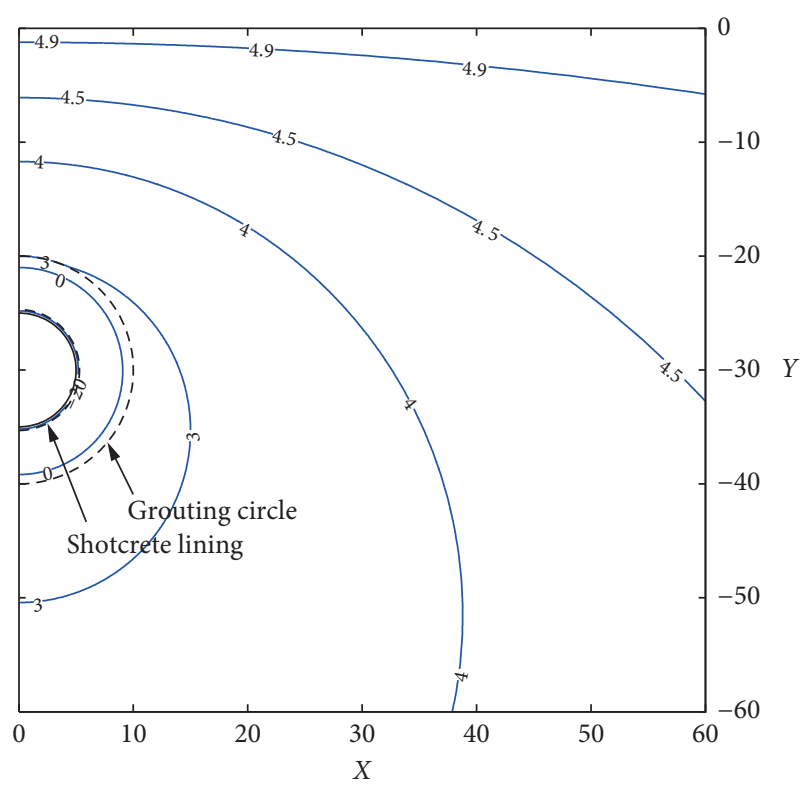

FIGURE 8: The water head distribution in case IV.

Working condition 4: Keep the pore pressure of measuring point 6 at $2.5 \mathrm{kpa}$, measure the steady flow rate of the outlet and pore pressure of other measuring points.

5.4. Test Results. As shown in Table 4, the results show that the two trends are consistent. The numerical errors are between $-2 \%$ and $16 \%$, which may be due to the inadequate accuracy of the test.

The water pressures at all points measured under various working conditions are shown in Figure 16. Compared with the theoretical calculation results in this paper, the results show that except for the slightly larger deviation of pore water pressures at 6 observation points, the consistency of the two points is good.

\section{Comparison with Other Analytical Solution}

Harr obtained an analytical solution in 1962, which is widely accepted to predict the water inflow of tunnels without lining in homogeneous, isotropic semi-infinite aquifers. When the permeability coefficient of grouting ring and shotcrete lining is exactly the same as that of the original stratum, the model in this paper can be degenerated into Harr model. That is to say, in formula (13), when $k_{l}=k_{g}=k_{s}=k$, it can be simplified as

$$
\begin{aligned}
q & =\frac{2 \pi k_{l} k_{g} k_{s}\left(H_{R}-H_{0}\right)}{k_{g} k_{s} \ln \left(r_{B 0} r_{A l} / r_{A 0} r_{B l}\right)+k_{l} k_{s} \ln \left(r_{B l} r_{A g} / r_{A l} r_{B g}\right)+k_{l} k_{g} \ln \left(r_{B g} / r_{A g}\right)}, \\
& =\frac{2 \pi k\left(H_{R}-H_{0}\right)}{\ln \left(r_{B 0} / r_{A 0}\right)} .
\end{aligned}
$$




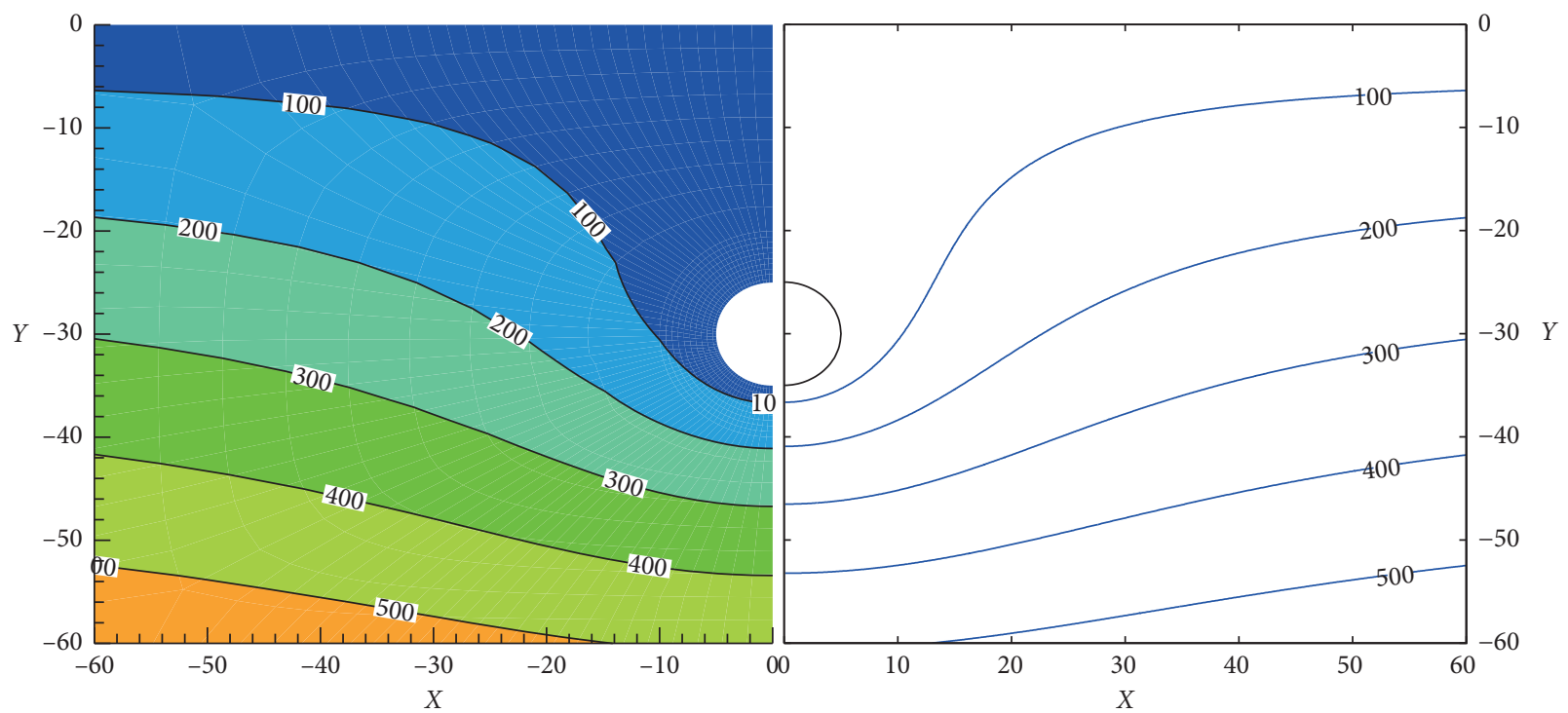

FIgURE 9: Comparison of water pressure distribution between the solution presented in this paper (on the right) and ABAQUS numerical solution (on the left) in case I.
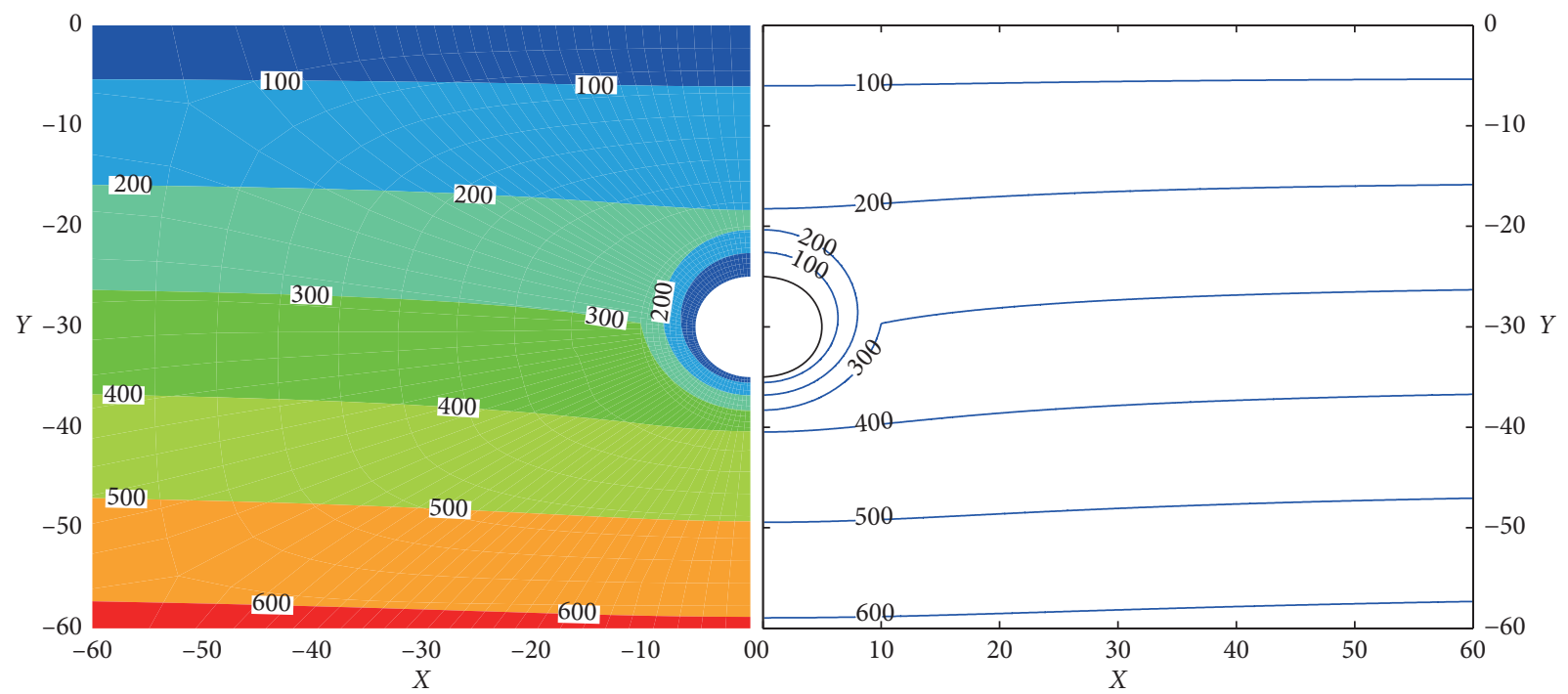

FIGURE 10: Comparison of water pressure distribution between the solution presented in this paper (on the right) and ABAQUS numerical solution (on the left) in case II.

Formula (16) turns out to be the Harr solution. It can be seen that the solution in this paper is the expansion of Harr solution while it has a wider scope of application.

\section{Parametric Analysis}

7.1. Influences of Grouting Ring Parameters. Grouting is one of the main methods of groundwater control in tunnel by drilling and blasting method. How to select the parameters of grouting ring is very important for design work. Exploring its influence law can provide guidance for design. Next, the influence of grouting circle permeability coefficient (reflecting grouting quality) and grouting circle thickness on tunnel water inflow and water pressure are analyzed.

7.1.1. Permeability Coefficient of Grouting Ring. According to formulas (13) and (15), the partial differential of tunnel water inflow and water pressure on the permeability coefficient of grouting ring is obtained, respectively. 


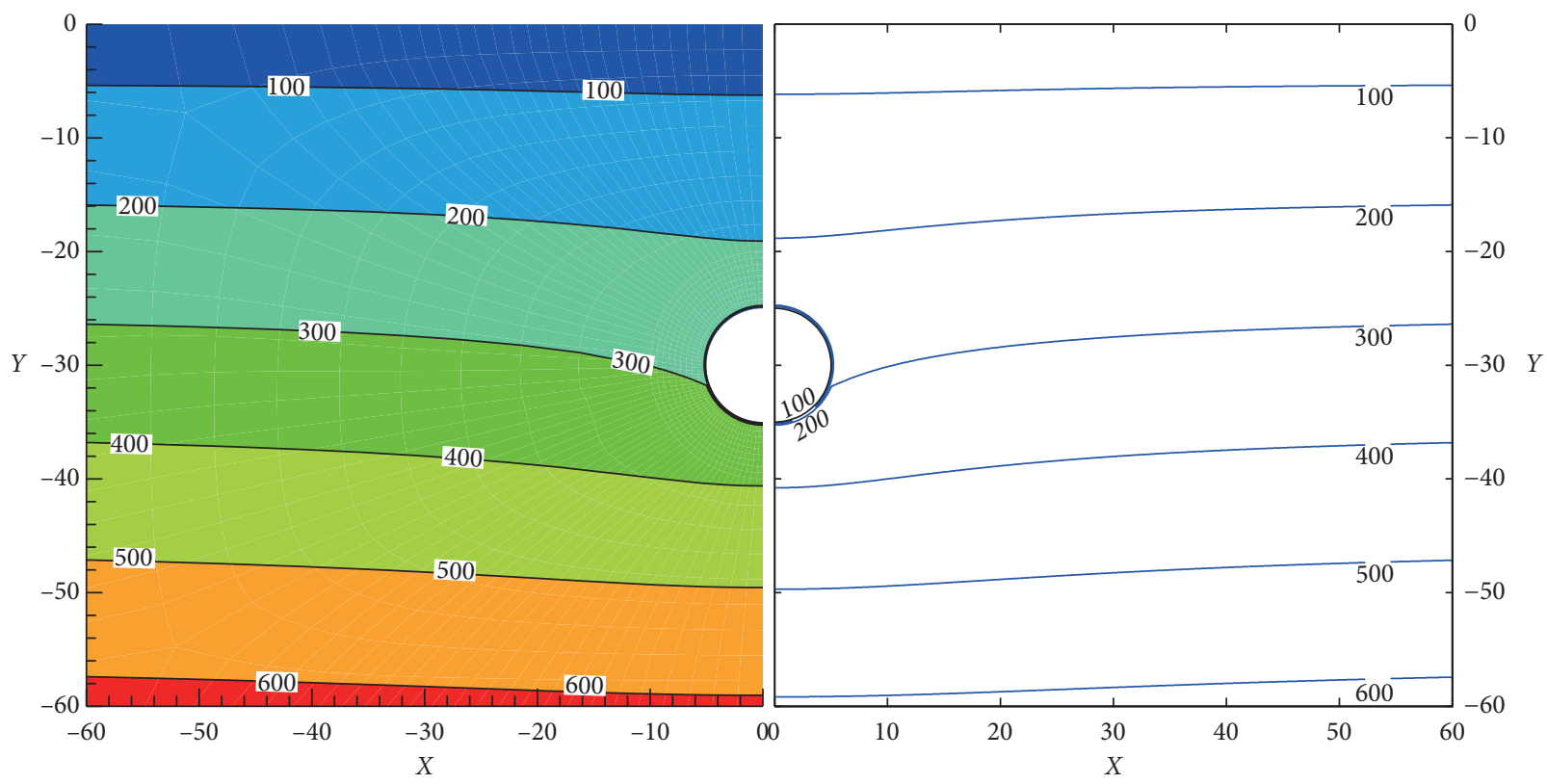

FIGURE 11: Comparison of water pressure distribution between the solution presented in this paper (on the right) and ABAQUS numerical solution (on the left) in case III.

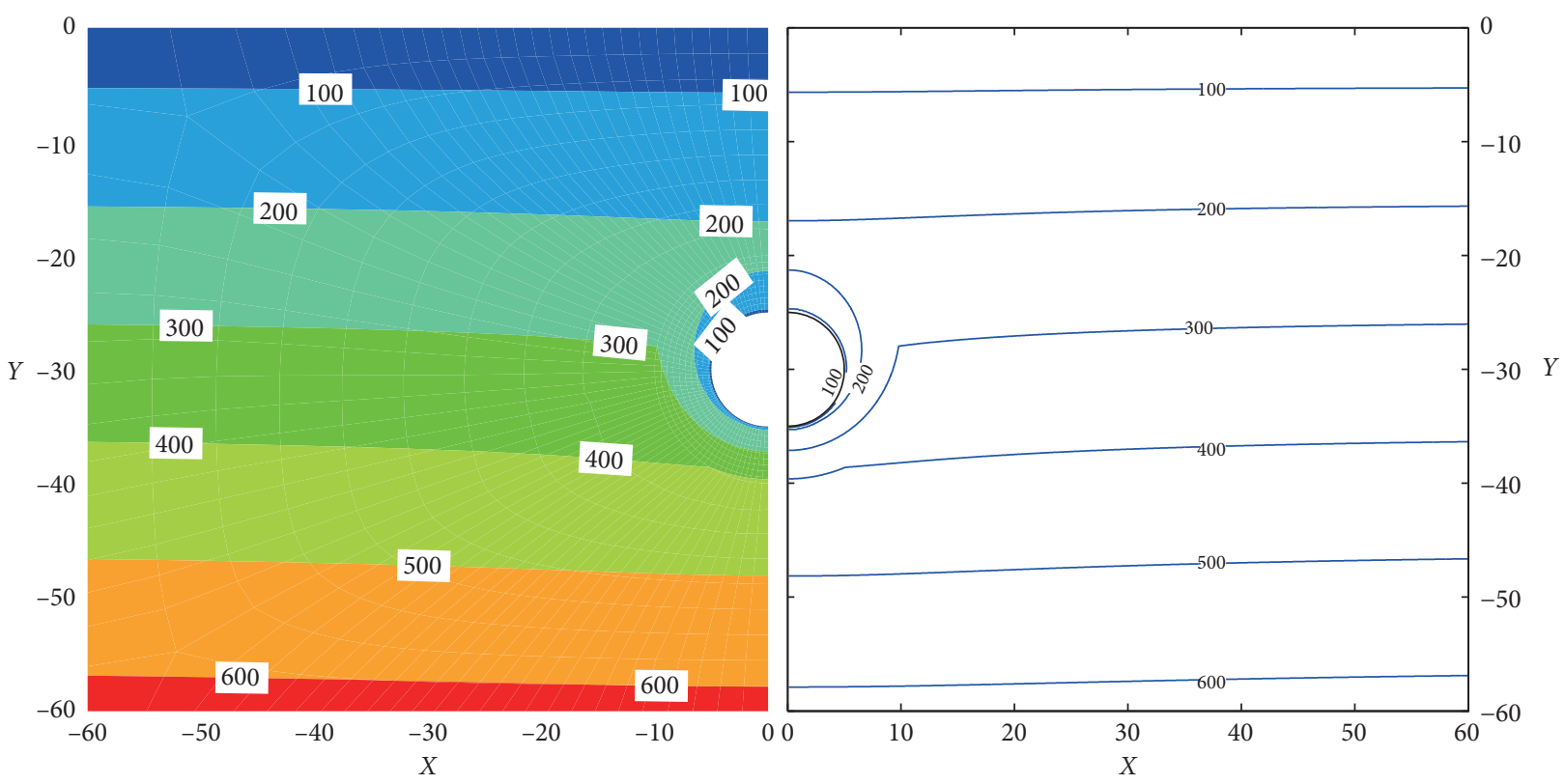

FIGURE 12: Comparison of water pressure distribution between the solution presented in this paper (on the right) and ABAQUS numerical solution (on the left) in case IV.

$$
\begin{aligned}
& \frac{\partial q}{\partial k_{g}}=\frac{2 \pi k_{l}^{2} k_{s}^{2}\left(H_{R}-H_{0}\right) \ln r_{B l} r_{A g} / r_{A l} r_{B g}}{\left(k_{g} k_{s} \ln \left(r_{B 0} r_{A l} / r_{A 0} r_{B l}\right)+k_{l} k_{s} \ln \left(r_{B l} r_{A g} / r_{A l} r_{B g}\right)+k_{l} k_{g} \ln \left(r_{B g} / r_{A g}\right)\right)^{2}}>0, \\
& \frac{\partial p_{g}}{\partial k_{g}}=\gamma_{w} \frac{\partial H_{g}}{\partial k_{g}}=-\gamma_{w} \frac{q}{2 \pi k_{g}^{2}} \ln \frac{r_{B l} r_{A g}}{r_{A l} r_{B g}}<0 .
\end{aligned}
$$




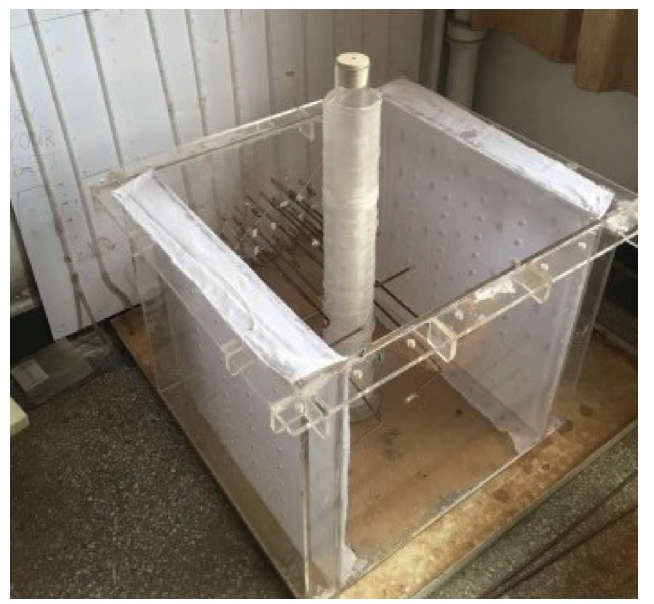

(a)

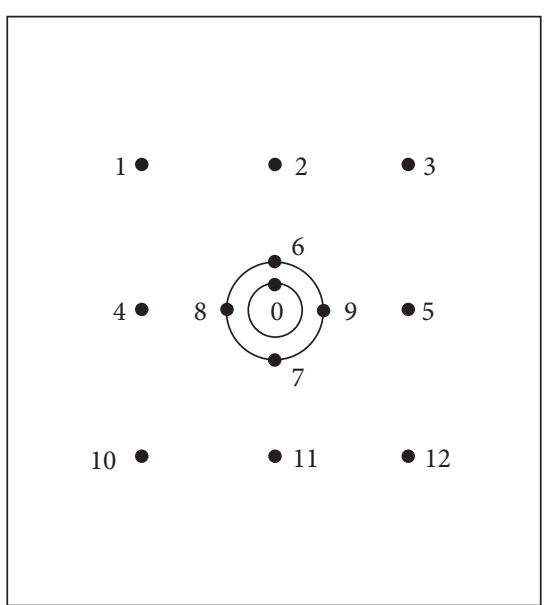

(b)

FIgURE 13: Layout of laboratory test box and water pressure measuring points.

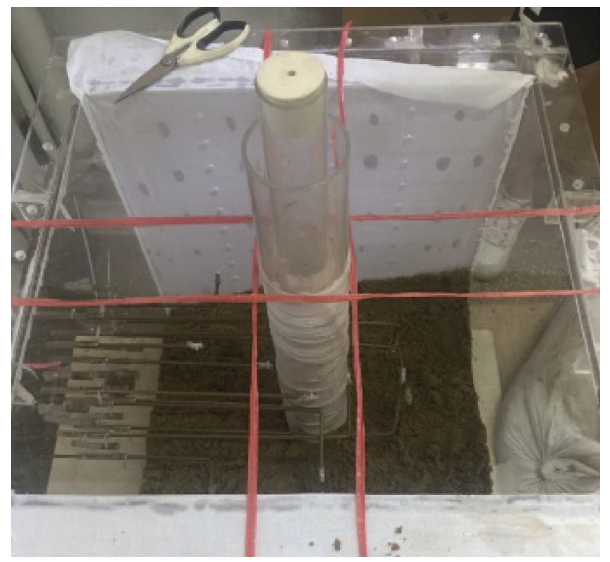

(a)

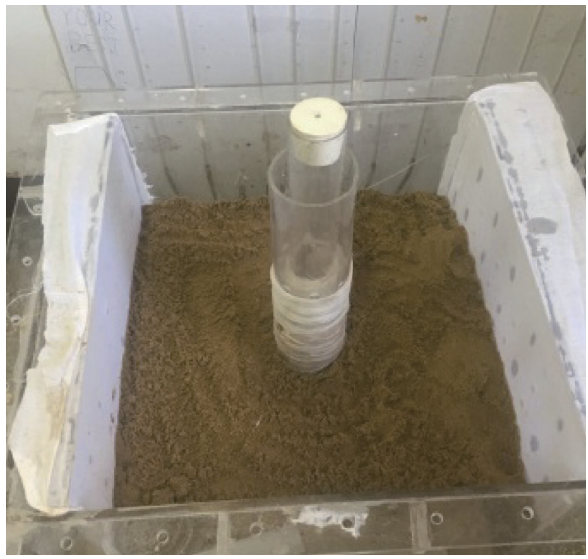

(b)

FIGURE 14: Sand filling.

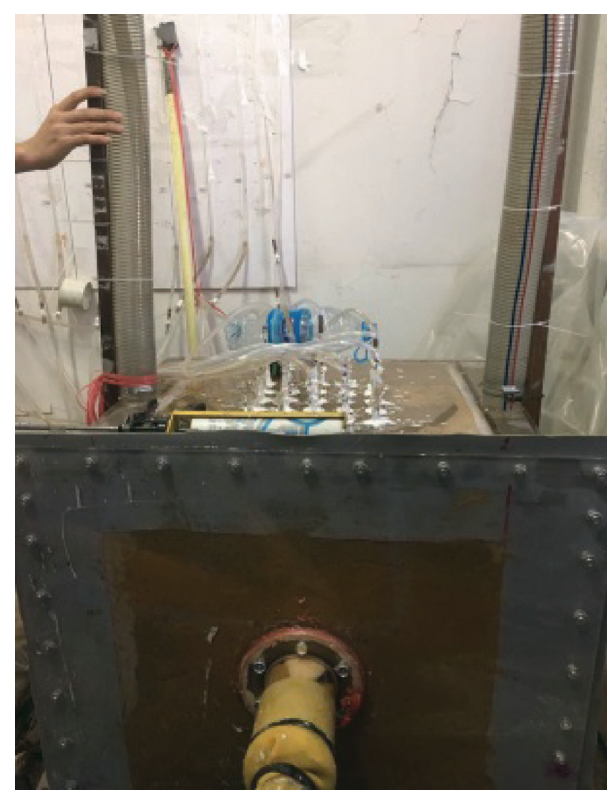

Figure 15: Test process. 
TABLE 4: Contrast table of tunnel water inflow $\left(\mathrm{m}^{3} / \mathrm{d}\right)$.

\begin{tabular}{lcccc}
\hline & Working condition 1 & Working condition 2 & Working condition 3 & Working condition 4 \\
\hline Test & 2.76 & 4.68 & 5.40 & 9.22 \\
This paper & 2.37 & 4.05 & 5.50 & 8.60 \\
Errors & $16 \%$ & $16 \%$ & $-2 \%$ & $7 \%$ \\
\hline
\end{tabular}
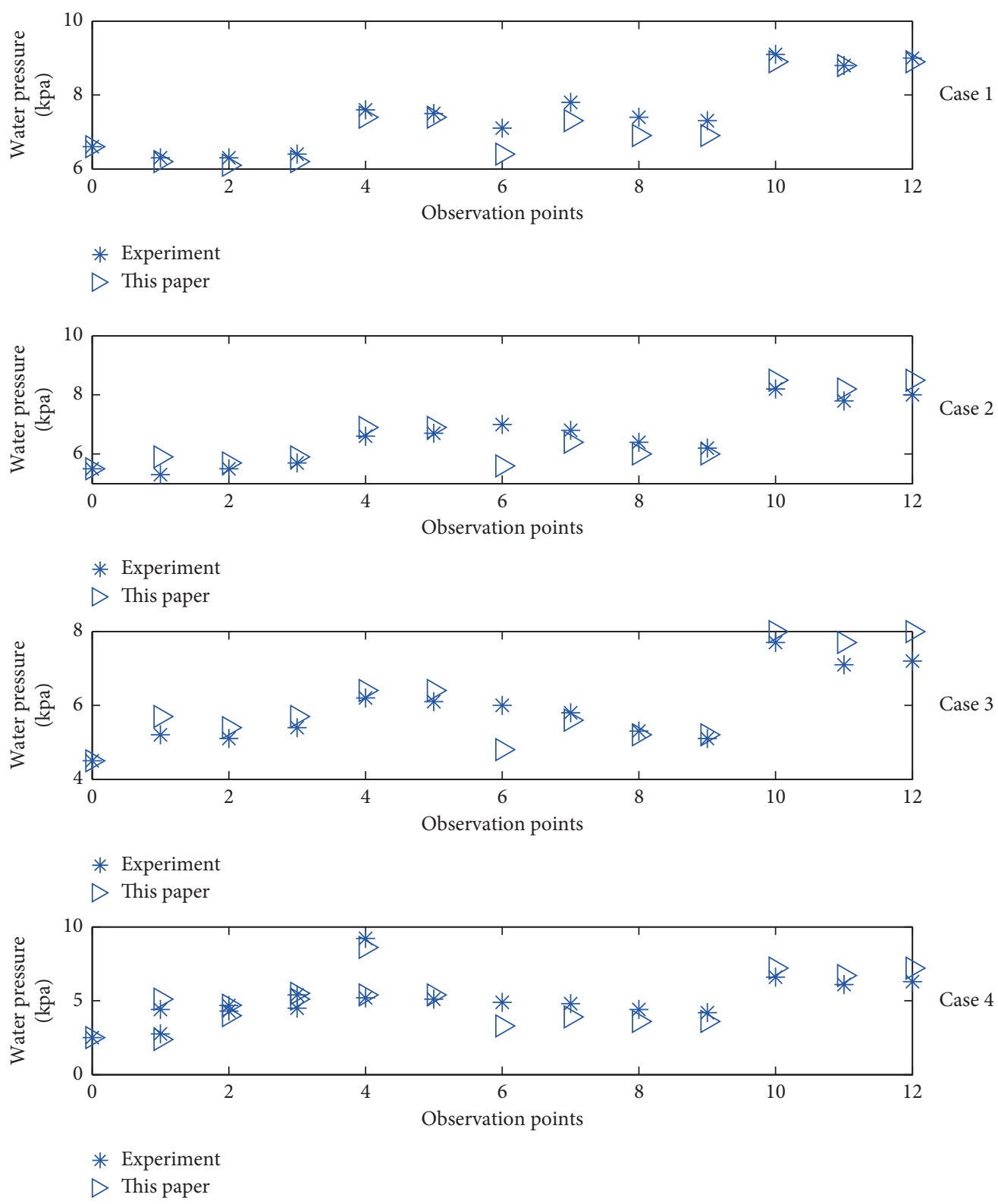

FIGURE 16: Water pressure comparison diagram at the observation points.

It can be seen that the tunnel water inflow is an increasing function of the permeability coefficient of grouting ring, and the water pressure on grouting ring is a decreasing function of the permeability coefficient of grouting ring. From engineering understanding, the larger the permeability coefficient of grouting ring (the worse the grouting quality) is, the larger the tunnel water inflow will be and the smaller the water pressure the grouting ring needs to bear. Meanwhile, the smaller the permeability coefficient of grouting ring (the better the grouting quality), the smaller the water inflow of tunnel. At the same time, the greater the water pressure, the grouting ring needs to bear.

To further verify, the permeability coefficient of grouting ring is adjusted for the working condition IV in Section 4.1, while other parameters remain unchanged. The effect of 
permeability coefficient on tunnel water inflow and water pressure on grouting ring is shown in Figure 17. It can be seen that the trend of change in the diagram is consistent with the analysis mentioned above.
7.1.2. Thickness of Grouting Ring. According to formulas (13) and (15), the partial differential of tunnel water inflow and water pressure on grouting ring thickness is obtained, respectively.

$$
\begin{aligned}
\frac{\partial q}{\partial r_{g}} & =-\frac{2 \pi k_{l}^{2} k_{g} k_{s}\left(H_{R}-H_{0}\right)\left(k_{s}-k_{g}\right)}{\left(k_{g} k_{s} \ln \left(r_{B 0} r_{A l} / r_{A 0} r_{B l}\right)+k_{l} k_{s} \ln \left(r_{B l} r_{A g} / r_{A l} r_{B g}\right)+k_{l} k_{g} \ln \left(r_{B g} / r_{A g}\right)\right)^{2} r_{A g}}<0, \\
\frac{\partial p_{g}}{\partial r_{g}} & =\gamma_{w} \frac{q}{2 \pi k_{g} r_{A g}}>0 .
\end{aligned}
$$

It can be seen that the water inflow of tunnel is a decreasing function of the thickness of grouting ring, and the water pressure on grouting ring is an increasing function of the thickness of grouting ring. From engineering understanding, the greater the thickness of grouting ring, the smaller the water inflow of tunnel. At the same time, the greater the water pressure the grouting ring needs to bear; Conversely, the smaller the thickness of the grouting ring in the tunnel, the greater the amount of water flowing in the tunnel, and the smaller the water pressure that the grouting ring needs to bear.

In order to further verify, the thickness of grouting ring is adjusted for the working condition IV in Section 4.1, while other parameters remain unchanged. The influence of grouting ring thickness on tunnel water inflow and water pressure on grouting ring is shown in Figure 18. It can be seen that the trend of change in the diagram is consistent with the analysis mentioned above.

\subsection{Influences of Primary Lining Parameters}

7.2.1. Permeability Coefficient of Primary Lining. According to formulas (13) and (15), the partial differential of tunnel water inflow and water pressure on the permeability coefficient of primary lining is obtained, respectively.

$$
\begin{aligned}
& \frac{\partial q}{\partial k_{l}}=\frac{2 \pi k_{g}{ }^{2} k_{s}^{2}\left(H_{R}-H_{0}\right) \ln r_{B 0} r_{A l} / r_{A 0} r_{B l}}{\left(k_{g} k_{s} \ln \left(r_{B 0} r_{A l} / r_{A 0} r_{B l}\right)+k_{l} k_{s} \ln \left(r_{B l} r_{A g} / r_{A l} r_{B g}\right)+k_{l} k_{g} \ln \left(r_{B g} / r_{A g}\right)\right)^{2}}>0, \\
& \frac{\partial p_{l}}{\partial k_{l}}=-\gamma_{w} \frac{q}{2 \pi k_{l}^{2}} \ln \frac{r_{B 0} r_{A l}}{r_{A 0} r_{B l}}<0 .
\end{aligned}
$$

It can be seen that the tunnel water inflow is an increasing function of the permeability coefficient of primary lining, and the water pressure on primary lining is a decreasing function of the permeability coefficient of primary lining. From engineering understanding, the larger the permeability coefficient of primary lining is, the larger the tunnel water inflow will be. At the same time, the smaller the water pressure the primary lining needs to bear. Meanwhile, the smaller the permeability coefficient of primary lining, the smaller the water inflow of tunnel. At the same time, the greater the water pressure the primary lining needs to bear.

To further verify, the permeability coefficient of primary lining is adjusted for the working condition IV in Section 4.1, while other parameters remain unchanged. The effect of permeability coefficient on tunnel water inflow and water pressure on primary lining is shown in Figure 19. It can be seen that the trend of change in the diagram is consistent with the analysis mentioned above.

7.2.2. Thickness of Primary Lining. According to formulas (13) and (15), the partial differential of tunnel water inflow and water pressure on primary lining thickness is obtained, respectively.

$$
\begin{aligned}
\frac{\partial q}{\partial r_{l}} & =-\frac{2 \pi k_{l} k_{g} k_{s}^{2}\left(H_{R}-H_{0}\right)\left(k_{g}-k_{l}\right)}{\left(k_{g} k_{s} \ln \left(r_{B 0} r_{A l} / r_{A 0} r_{B l}\right)+k_{l} k_{s} \ln \left(r_{B l} r_{A g} / r_{A l} r_{B g}\right)+k_{l} k_{g} \ln \left(r_{B g} / r_{A g}\right)\right)^{2} r_{A l}}<0 \\
\frac{\partial p_{l}}{\partial r_{l}} & =\gamma_{w} \frac{q}{2 \pi k_{l} r_{A l}}>0 .
\end{aligned}
$$




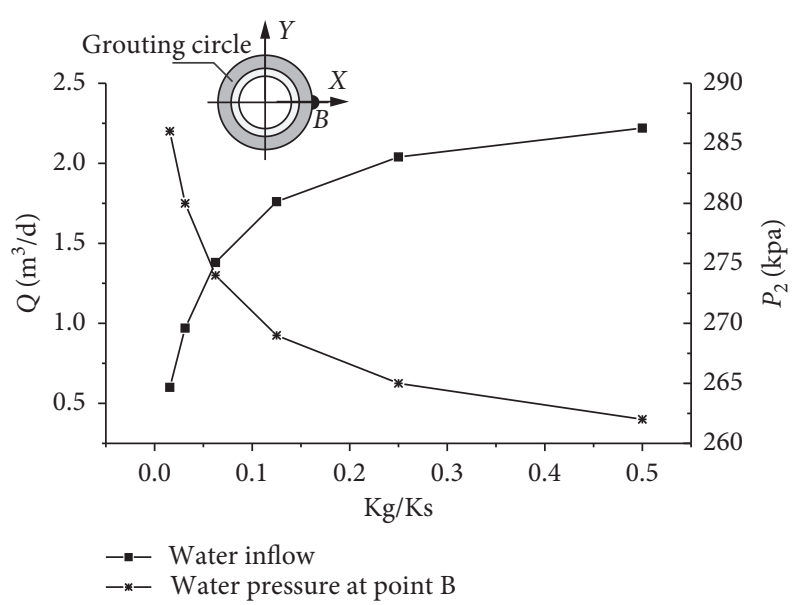

FIgURE 17: The influence of grouting circle permeability coefficient on tunnel water inflow and water pressure borne by grouting circle.

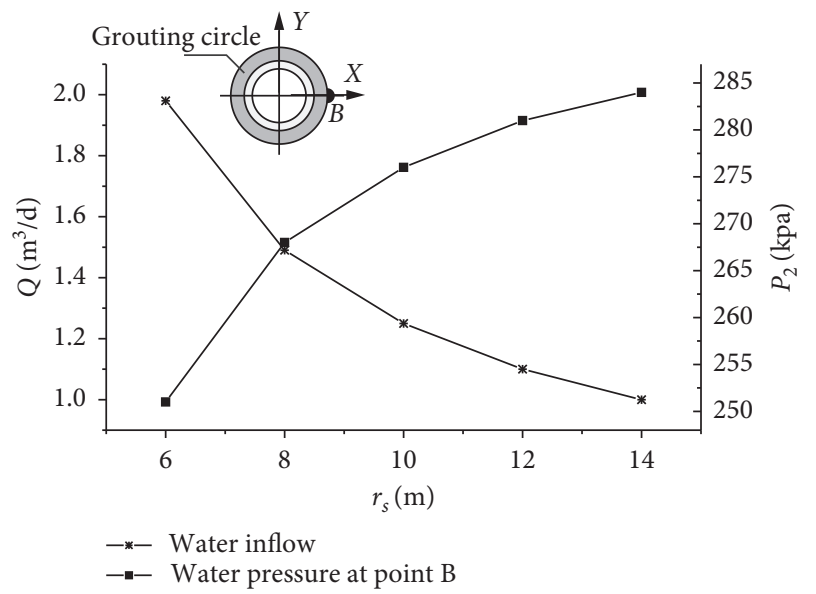

FIGURE 18: The influence of grouting ring thickness on tunnel water inflow and water pressure borne by grouting ring.

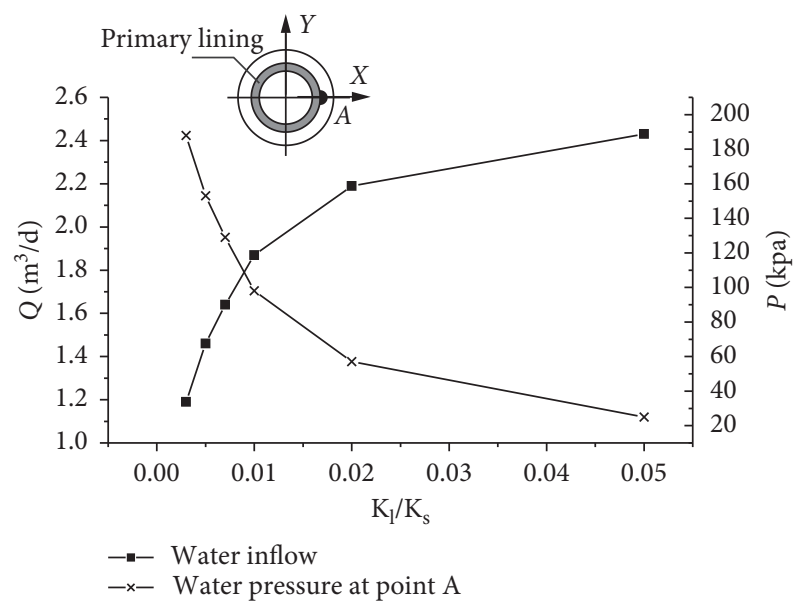

FIgURE 19: The influence of primary lining permeability coefficient on tunnel water inflow and water pressure borne by primary lining.

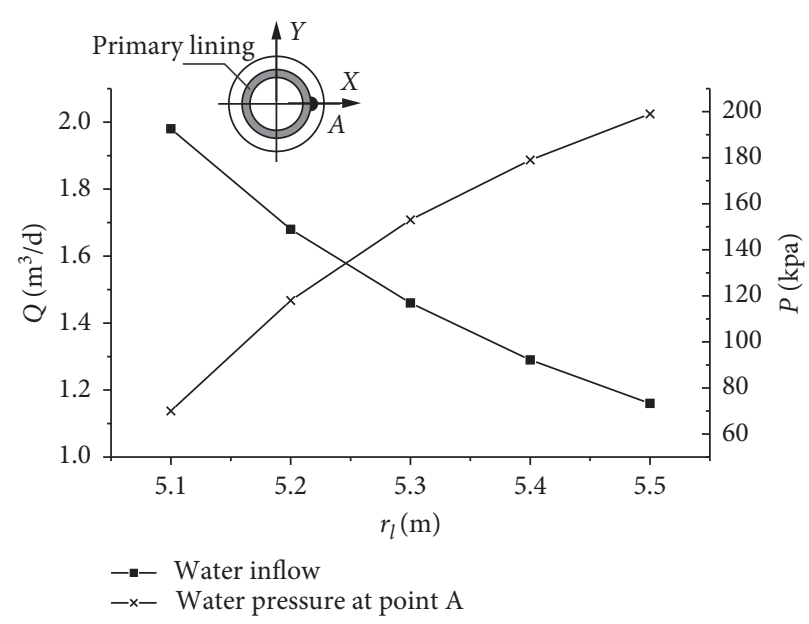

FIgURE 20: The influence of primary lining thickness on tunnel water inflow and water pressure borne by primary lining.

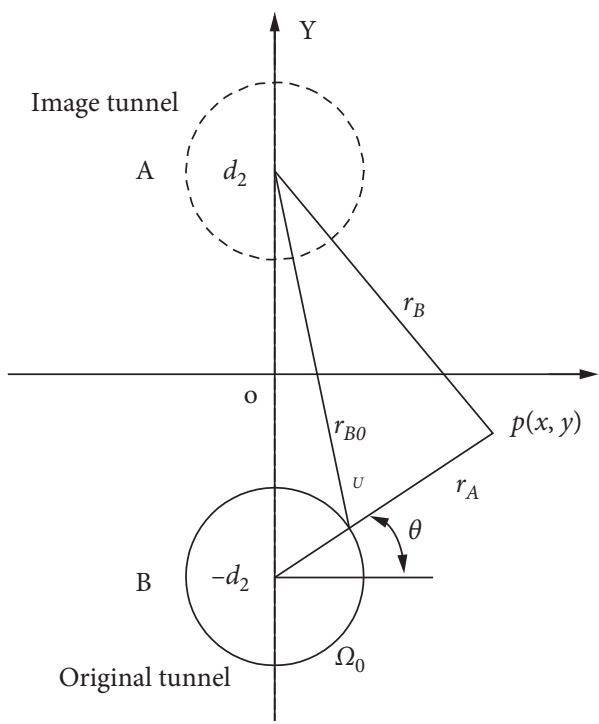

FIgURE 21: Parametric geometric diagram.

It can be seen that the water inflow of tunnel is a decreasing function of the thickness of primary lining, and the water pressure on primary lining is an increasing function of the thickness of primary lining. From engineering understanding, the greater the thickness of primary lining, the smaller the water inflow of tunnel. At the same time, the greater the water pressure the primary lining needs to bear; Conversely, the smaller the thickness of the primary lining in the tunnel, the greater the amount of water flowing in the tunnel, and the smaller the water pressure that the primary lining needs to bear.

In order to further verify, the thickness of primary lining is adjusted for the working condition IV in Section 4.1, while other parameters remain unchanged. The influence of primary lining thickness on tunnel water inflow and water pressure on primary lining is shown in Figure 20. It can be 
TABle 5: Parameters calculating table.

\begin{tabular}{lcr}
\hline Points & Coordinate & Parameter values \\
\hline$P$ & $(x, y)$ & $r_{A 0}=|A U|$ \\
$A$ & $\left(0,-d_{2}\right)$ & $r_{B 0}=|B U|$ \\
$B$ & $\left(0, d_{2}\right)$ & $r_{A l}=|A V|$ \\
$U$ & $r_{B l}=|B V|$ \\
$V$ & $\left(r_{0} \cos \theta,-d_{2}+r_{0} \sin \theta\right)$ & $r_{A g}=|A W|$ \\
$W$ & $\left(r_{l} \cos \theta,-d_{2}+r_{l} \sin \theta\right)$ & $r_{B g}=|B W|$ \\
& $\left(r_{g} \cos \theta,-d_{2}+r_{g} \sin \theta\right)$ & $r_{A}=|A P|$ \\
& & $r_{B}=|B P|$ \\
& & $\theta=\arctan \left(y+d_{2} / x\right)$ \\
\hline
\end{tabular}

seen that the trend of change in the diagram is consistent with the analysis mentioned above.

7.3. Physical Significance and Calculation of Parameters. For application purpose, the following is a further explanation of the calculation of the parameters in the formulas mentioned above.

As shown in Figure 21, the physical meaning of $r_{A 0}$ is the distance between the center of tunnel $A$ and the boundary $\Omega_{0}$ of tunnel $A$. It is a fixed value, i.e., the radius of tunnel $A$.

The physical meaning of $r_{B 0}$ is the distance between the center of tunnel $B$ and the boundary $\Omega_{0}$ of tunnel $A$. Because $\Omega_{0}$ is a circle, not a point, the value of $r_{B 0}$ needs to be determined according to the specific location of the calculated point. As shown in Figure 17, the water head of a point $P(x, y)$ is calculated according to equation (14), and $r_{B 0}$ equals the distance of segment $|B U|$. Point $U$ is the intersection of line $A P$ and the boundary $\Omega_{0}$ of tunnel $A$. Point $B$ is the center of tunnel $B$.

Similarly, point $V$ is the intersection of line $A P$ and the outer boundary of tunnel shotcrete lining; point $W$ is the intersection of line $A P$ and the outer boundary of tunnel grouting ring. The calculation of relevant parameters is shown in Table 5.

\section{Conclusions}

In this paper, theoretical analysis and experimental study on seepage in underwater tunnel by drilling and blasting method are carried out. The conclusions and suggestions are as follows:

(1) The water inflow of an underwater tunnel can be calculated according to equation (13), and the total head of each point in the seepage field can be calculated according to equation (14). This conclusion can be used as a reference for the prediction of water inflow and the calculation of water load in tunnel structure.

(2) The better the grouting effect of grouting ring and primary lining (the smaller the permeability coefficient of grouting ring and primary lining) or the bigger the thickness of grouting ring and primary lining, the less water inflow of tunnel can be effectively reduced. At the same time, the water load on grouting ring and primary lining will increase. The relationship between them should be properly balanced in design.

\section{List of Symbols}

$\phi:$

$\phi_{A}, \phi_{B}$ :

$\phi_{1}, \phi_{A 1}, \phi_{B 1}:$

$\phi_{2}, \phi_{A 2}, \phi_{B 2}:$

The potential function of water head The potential function of water head of tunnel A and tunnel B, respectively

The potential function of water head in primary lining area

$\phi_{3}, \phi_{A 3}, \phi_{B 3}$ : The potential function of water head in grouting circle area

$\phi_{3}, \phi_{A 3}, \phi_{B 3}$ : The potential function of water head in surrounding ground area

$\phi_{\Omega 0}:$

$\phi_{\Omega l}:$ The potential function of water head along the inner boundary of primary lining The potential function of water head on the contact surface between the primary lining and the grouting circle

$\phi_{\Omega g}: \quad$ The potential function of water head on the contact surface between the grouting circle and the surrounding ground

$\phi_{\Omega R}$ : The potential function of water head at the ground surface

$r, \theta: \quad$ Polar coordinates

$x, y: \quad$ Rectangular coordinates

$r_{A}, r_{B}: \quad$ The distance from the center of tunnel A and tunnel $\mathrm{B}$ to the calculation point

$r_{A 0}, r_{B 0}:$

$r_{A l}, r_{B l}:$

$r_{A g}, r_{B g}:$

$r_{A R}, r_{B R}:$

$k, k_{l}, k_{g}, k_{s}:$ The distance between the center of tunnel A (tunnel B) and the boundary $\Omega_{0}$ of tunnel A The distance between the center of tunnel A (tunnel B) and the boundary $\Omega_{l}$ of tunnel A The distance between the center of tunnel A (tunnel B) and the boundary $\Omega_{g}$ of tunnel A The distance between the center of tunnel A (tunnel B) and the boundary $\Omega_{R}$ of tunnel A Permeability

$H, H_{l}, H_{g}, H_{s}$ : The water head on the boundary

$\mathrm{H}, \mathrm{H}_{1}, \mathrm{H}_{2}, \mathrm{H}_{3}$ : The water head in different areas

$P, P_{1}, P_{2}, P_{3}$ : The water pressure in different areas

$q, Q:$ The water inflow and the quantity of seepage

$\gamma_{w}$ : Unit weight of water

$C, C_{1}, C_{2}, C_{3}$ : Parameters determined by boundary conditions.

\section{Data Availability}

The data used to support the findings of this study are available from the corresponding author upon request.

\section{Conflicts of Interest}

The authors declare no conflicts of interest.

\section{Acknowledgments}

This research was funded by the National Natural Science Foundation of China (U1765204 and 41772340). 


\section{References}

[1] M. E. Harr, "Groundwater and Seepage," 1962.

[2] S. Lei, "An analytical solution for steady flow into a ttonnel," Ground Water, vol. 37, no. 1, pp. 23-26, 1999.

[3] R. E. Goodman, "Ground water inflows during tunnel driving," Bulletin of Engineering Geology and the Environment, vol. 2, pp. 35-36, 1965.

[4] L. Zhang and J. A. Franklin, "Prediction of water flow into rock tunnels: an analytical solution assuming an hydraulic conductivity gradient," International Journal of Rock Mechanics and Mining Sciences \& Geomechanics Abstracts, vol. 30, no. 1, pp. 37-46, 1993.

[5] M. El Tani, "Water inflow into tunnels," in Proceedings of the World Tunnel Congress ITA-AITES 1999, Oslo, Norway, 1999.

[6] M. El Tani, "Circular tunnel in a semi-infinite aquifer," Tunnelling and Underground Space Technology, vol. 18, no. 1, pp. 49-55, 2003.

[7] D. Kolymbas and P. Wagner, "Groundwater ingress to tunnels-the exact analytical solution," Tunnelling and Underground Space Technology, vol. 22, no. 1, pp. 23-27, 2007.

[8] K.-H. Park, A. Owatsiriwong, and J.-G. Lee, "Analytical solution for steady-state groundwater inflow into a drained circular tunnel in a semi-infinite aquifer: a revisit," Tunnelling and Underground Space Technology, vol. 23, no. 2, pp. 206209, 2008.

[9] K.-H. Park, J.-G. Lee, and A. Owatsiriwong, "Seepage force in a drained circular tunnel: an analytical approach," Canadian Geotechnical Journal, vol. 45, no. 3, pp. 432-436, 2008.

[10] M. Huangfu, M.-S. Wang, Z.-S. Tan, and X.-Y. Wang, "Analytical solutions for steady seepage into an underwater circular tunnel," Tunnelling and Underground Space Technology, vol. 25, no. 4, pp. 391-396, 2010.

[11] S.-Y. Yang and H.-D. Yeh, "A closed-form solution for a confined flow into a tunnel during progressive drilling in a multi-layer groundwater flow system," Geophysical Research Letters, vol. 34, no. 7, p. L07405, 2007.

[12] G. Fernandez and J. Moon, "Excavation-induced hydraulic conductivity reduction around a tunnel-part 1: guideline for estimate of ground water inflow rate," Tunnelling and Underground Space Technology, vol. 25, no. 5, pp. 560-566, 2010.

[13] J.-C. Maréchal, S. Lanini, B. Aunay, and P. Perrochet, "Analytical solution for modeling discharge into a tunnel drilled in a heterogeneous unconfined aquifer," Groundwater, vol. 52, no. 4, pp. 597-605, 2013.

[14] K. Su, An Analytical Method for Groundwater Inflow into a Drained Circular Tunnel, Ground Water, New York, NY, USA, 2017.

[15] H. Ying, C. Zhu, and X. Gong, "Tide-induced hydraulic response in a semi-infinite seabed with a subaqueous drained tunnel," Acta Geotechnica, vol. 13, no. 1, pp. 149-157, 2018.

[16] M. R. Zareifard, "An analytical solution for design of pressure tunnels considering seepage loads," Applied Mathematical Modelling, vol. 62, pp. 62-85, 2018.

[17] C. Butscher, "Steady-state groundwater inflow into a circular tunnel," Tunnelling and Underground Space Technology, vol. 32, pp. 158-167, 2012.

[18] H. Farhadian, A. Nikvar Hassani, and H. Katibeh, "Groundwater inflow assessment to karaj water conveyance tunnel, northern Iran," KSCE Journal of Civil Engineering, vol. 21, no. 6, pp. 2429-2438, 2016.

[19] H. Farhadian and H. Katibeh, "New empirical model to evaluate groundwater flow into circular tunnel using multiple regression analysis," International Journal of Mining Science and Technology, vol. 27, no. 3, pp. 415-421, 2017.

[20] H. Farhadian and H. Katibeh, "Effect of model dimesion in numerical simulation on assessment of water inflow to tunnel in discontinues rock, World Academy of Science, Engineering and Technology," International Journal of Environmental, Ecological, Geological and Geophysical Engneering, vol. 9, no. 4, pp. 257-260, 2015.

[21] N. Hassani, "Numerical analysis of steady-state groundwater inflow into Tabriz line 2 metro tunnel, northwestern Iran, with special consideration of model dimensions," Bulletin of Engineering Geology and the Environment, vol. 75, pp. 16171627, 2016.

[22] K. Holmoy and H. B. Nilsen, "Significance of geological parameters for predicting water inflow in hard rock tunnels," Rock Mechanics and Rock Engineering, vol. 47, no. 3, pp. 853-868, 2014.

[23] H. Farhadian, "Empirical model for estimating groundwater flow into tunnel in discontinuous rock masses," Environmental Earth Sciences, vol. 75, p. 471, 2016.

[24] J. Font-Capó, E. Vázquez-Suñé, J. Carrera, D. Martí, R. Carbonell, and A. Pérez-Estaun, "Groundwater inflow prediction in urban tunneling with a tunnel boring machine (TBM)," Engineering Geology, vol. 121, pp. 46-54, 2011.

[25] K. Karlsrud, "Water Control When Tunnelling under Urban Areas in the Olso Region," NFF Publication No. 12, NFF, 2001. 